\title{
Dexamethasone and sex regulate placental glucocorticoid receptor isoforms in mice
}

\author{
James S M Cuffe ${ }^{1,2,3}$, Zarqa Saif ${ }^{3}$, Anthony V Perkins', Karen M Moritz ${ }^{2}$ and \\ Vicki L Clifton ${ }^{3}$ \\ 'School of Medical Science and Menzies Health Institute Queensland, Griffith University, Southport, \\ Queensland, Australia \\ ${ }^{2} 5$ chool of Biomedical Sciences, and Child Health Research Centre, The University of Queensland, St \\ Lucia, Queensland, Australia \\ ${ }^{3}$ Mater Research Institute, Translational Research Institute, University of Queensland, Woolloongabba, \\ Queensland, Australia
}

Correspondence should be addressed to $\mathrm{V}$ L Clifton Email

Vicki.clifton@mater.uq. edu.au

\begin{abstract}
Maternal dexamethasone exposure in the mouse impairs placental development and programs adult disease in a sexually dimorphic manner. Glucocorticoids bind to different glucocorticoid receptor (GR) isoforms to regulate gene transcription and cellular signaling. We hypothesized that sexually dimorphic placental responses to glucocorticoids are due to differences in GR isoforms present in the placenta. Pregnant C57BI6 mice were exposed to saline or dexamethasone from E12.5 until E14.5 $(1 \mu \mathrm{g} / \mathrm{kg} / \mathrm{h})$ before the collection of placentae. Cytoplasmic and nuclear protein fractions were extracted from placentae of male and female fetuses for Western blot analysis of GR isoforms. Eight known isoforms of the GR were detected in the mouse placenta including the translational isoforms $G R \alpha-A, B, C$ and $D 1-3$ and the splice variants GRA and GRP. The expression of GRA, GRP and each of the GR $\alpha$ isoforms were altered by dexamethasone in relation to fetal sex and cellular location. Placentae of female fetuses had higher GR $\alpha-A$ and GRP expression in the cytoplasm than males, and GR $\alpha-C$ was more highly expressed in the nucleus of females than that in males. Dexamethasone significantly increased the cytoplasmic expression of $\mathrm{GR} \alpha-\mathrm{A}$, but reduced the expression of $\mathrm{GR} \alpha-\mathrm{C}$ in placentae of males. Dexamethasone increased the expression of the GR $\alpha-C-$ regulated genes Sgk1 and BC/2/11, particularly in females. The cleaved caspase-3 staining in placental sections indicated $\mathrm{GR} \alpha-\mathrm{C}$ may mediate sex differences in dexamethasoneinduced apoptosis. These findings may underlie the sex-specific placental adaptations that regulate different growth profiles in males and females and different risks for programmed disease outcomes in offspring.
\end{abstract}

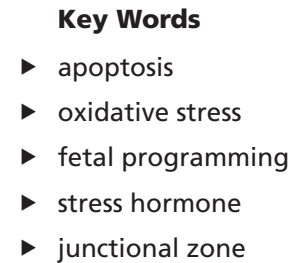

Journal of Endocrinology (2017) 234, 89-100

\section{Introduction}

Maternal glucocorticoid exposure programs offspring disease in a sexually dimorphic manner (O'Regan et al. 2004, Singh et al. 2012). This is in part due to sexually dimorphic placental responses to glucocorticoids during pregnancy, which mediate different fetal growth strategies (Audette et al. 2010, Clifton 2010, Eriksson et al. 2010, O'Connell et al. 2011, Vaughan et al. 2012). We have reported that maternal dexamethasone exposure in the

Published by Bioscientifica Ltd. 
mouse reduces weight in both male and female fetuses but reduces placental weight in female fetuses only (Cuffe et al. 2011). The reduction in placental weight of females was associated with a reduction in the size of the junctional zone but an increase in the glucocorticoid metabolizing enzyme hydroxysteroid 11- $\beta$-dehydrogenase isozyme 2 (hsd11b2). These female adaptations likely prevent the cardiovascular and renal dysfunction that develop only in male offspring (O'Sullivan et al. 2013).

Normal regulation of glucocorticoid signaling through intricate interactions with steroid receptors is vital for healthy development. While natural glucocorticoids (cortisol and corticosterone) can bind to both the glucocorticoid receptor (GR) and mineralocorticoid receptor (MR) to mediate cellular outcomes, synthetic glucocorticoids such as dexamethasone act exclusively by binding to the GR. The wide range of placental adaptations to glucocorticoids are likely to be in part due to the complex nature of GR signaling, which directly or indirectly regulates up to $20 \%$ of the genome (Galon et al. 2002). The GR is predominantly localized to the cytoplasm, bound within the GR complex containing proteins such as Hsp90, Hsp70, Fkpb51 and c-Src. Once glucocorticoids bind to the GR, the GR complex proteins are released allowing translocation to the nucleus for gene transcription/transactivation by binding to either positive or negative glucocorticoid-responsive elements (GREs) (Cain \& Cidlowski 2015).

The GR is encoded by the single gene, Nr3c1, which has at least 5 characterized splice variants (GR $\alpha$, GR $\beta$, GR $\gamma$, GRA and GRP). Each of these splice variants have 8 different initiation sites (A, B, C1, C2, C3, D1, D2 and D3) resulting in up to 40 potential GR isoforms (Cain \& Cidlowski 2015). Receptor isoforms regulate different downstream signaling pathways with GR $\beta$ thought to largely oppose the actions of GR $\alpha$-A. Furthermore, GRA and GRP lack a ligand-binding domain and so indirectly regulate glucocorticoid signaling by dimerizing with GR $\alpha$ A. GR $\alpha$-D isoforms are predominantly expressed in the nucleus where they can regulate gene transcription in the absence of a ligand, whereas GR $\alpha-C$ is thought to make cells more sensitive to GR-induced cell death (Oakley \& Cidlowski 2013) by regulating downstream genes such as Bcl2111 (Wu et al. 2013).

The human and guinea pig placenta express at least 8 different known GR isoforms (Saif et al. 2015, 2016). Multiple GR isoforms have been detected in mouse dendritic cells, but the GR isoforms in the mouse placenta are unknown. This study firstly aimed to investigate the pattern of GR isoforms in the mouse placenta of males and females and then examine if this was affected by maternal dexamethasone exposure. Secondly, we examined the relationship between the pattern of GR isoforms and markers of placental apoptosis. Glucocorticoids induce cell death via multiple pathways and while apoptosis is a normal part of placental development (Sharp et al. 2010), inappropriate placental apoptosis may regulate programmed disease outcomes (Fowden et al. 2008). Indeed, sex-specific placental apoptosis has been recently demonstrated in sheep exposed to dexamethasone (Braun et al. 2015) that develop impaired endocrine function in later life. We hypothesized that maternal dexamethasone would alter the expression of GR isoforms including $\mathrm{GR} \alpha-\mathrm{C}$ and result in glucocorticoid-regulated cell death in female placentae contributing to our reported sex differences in placental growth (Cuffe et al. 2011).

\section{Methods}

All animal experiments were approved by the University of Queensland Animal Ethics Committee (AEC approval number SBMS/355/09) and were conducted in accordance with the Australian Code of Practice for the Care and Use of Animals for Scientific Purposes. The breeding and treatment protocol used for the current study was as described previously (Cuffe et al. 2011, 2015, O'Sullivan et al. 2013). Briefly, at E12.5, pregnant mice were surgically implanted with an osmotic minipump containing either dexamethasone $(n=10,1 \mu \mathrm{g} / \mathrm{kg} / \mathrm{h})$ or saline $(n=10,0.9 \%)$. Animals were killed for placental collection at E14.5. Collected placentae were snap-frozen in liquid nitrogen for molecular analysis or fixed in $4 \%$ paraformaldehyde, embedded in paraffin and sectioned on a microtome for protein localization.

\section{GR isoform protein analysis}

Protein was extracted from $60 \mathrm{mg}$ of placental tissue using a cytosolic fractionation buffer as described previously (Saif et al. 2015). 1-2 placentas were selected at random from each litter such that when possible, all 10 litters were represented ( $n=8-12$ samples per sex, per treatment). When more than one placenta was used from a litter, then data were pooled for that litter. After centrifugation, the cytoplasmic fraction was collected and the pellet was suspended in nuclear extraction buffer for nuclear protein extraction. $60 \mu \mathrm{g}$ of total protein was loaded into 3-8\% Tris-acetate gradient polyacrylamide gels (Invitrogen, Life Technologies). Each nuclear protein extract was loaded

Published by Bioscientifica Ltd. 
adjacent to its cytosolic counterpart and males and females were loaded on separate gels. A cross gel calibrator sample was loaded onto blots of the same sex so that all samples belonging to the same sex could be compared between gels. Proteins were transferred onto PVDF membranes, which were incubated with the rabbit antihuman GR total antibody (1:1500, Bethyl Laboratories, Montgomery, TX, USA, Cat no. A303-491A) as previously described (Saif et al. 2015), before being incubated with an anti-rabbit secondary antibody (1:2500) for $1 \mathrm{~h}$. Positive control samples (human placenta, mouse kidney protein) and negative controls (pre-absorbed sample with GR control peptide supplied with antibody) were run on separate blots (data not shown). An anti-beta actin antibody was subsequently applied to each membrane (ACTB, 1:4000, Bethyl Laboratories, Cat no. A300491A) for $1 \mathrm{~h}$ and densitometric analysis was performed. All receptor isoform values were normalized to ACTB expression. ACTB protein expression was not affected by sex or dexamethasone exposure although expression levels differed between nuclear and cytoplasmic fractions (data not shown). For initial analysis in saline animals, protein concentrations for each GR isoform were normalized to the total GR expression (average of GR bands from within a protein sample). This allowed comparisons between GR isoforms within a sample. Protein expression differed significantly between isoforms, therefore, for subsequent analysis comparing the effects of dexamethasone on individual isoform expression, relative protein expression was used (relative to its matched saline control).

\section{Downstream transcript analysis}

mRNA levels of genes known to be regulated by specific GR isoforms were measured by QPCR. Total RNA was extracted from placental samples (7-11 placentas per sex, per treatment-samples selected as for Western blot analysis) and reverse transcribed as previously described (Cuffe et al. 2012). mRNA levels of Cpeb1 (GRo-D3), Sgk1 and Bcl2l11 (GRo-C), Ier3 (all isoforms) and Nfatc1 (Gro-A) (Lu \& Cidlowski 2005) were analyzed using kiqstartsybr primers from Sigma Life Science (primer pair 1 for all primer sets). In addition, mRNA levels of importin 7 (Ipo7, a ROS-regulated molecule important for GR nuclear translocation) and heat shock protein family A (Hsp70) member 1B (Hspa1b, a chaperone protein involved in regulating GR function) were measured. All qPCR reactions were analyzed using the 2-DDCT method compared to the geometric mean of two validated endogenous control genes (Rn18s and $A c t b)$ and normalized to the mean of the saline group of their own sex. The geometric mean of Rn18s and $A c t b$ was not affected by treatment or sex (saline male $16.12 \pm 1.285$, dexamethasone male $15.87 \pm 0.6245$, saline female $16.16 \pm 0.4246$ and dexamethasone female $16.19 \pm 0.5591)$.

\section{Immunohistochemistry}

Evidence of apoptosis was firstly assessed using immunohistochemical staining of cleaved caspase-3. Representative midline placental sections were deparaffinized in xylene and rehydrated through decreasing concentrations of ethanol ( $n=3$ per group). All slides were subjected to antigen retrieval in citrate buffer at $85^{\circ} \mathrm{C}$ and were immersed in $0.9 \%$ hydrogen peroxide to block endogenous peroxidase activity. Slides were blocked in $2 \%$ bovine serum albumin (BSA) and incubated with a cleaved caspase-3 primary antibody (1:200, Cell Signaling, \#9661) diluted in 1\% BSA or a no-antibody control for $4 \mathrm{~h}$ at $4^{\circ} \mathrm{C}$. Slides were incubated with a biotinylated anti-rabbit secondary antibody (ABC Vectastain Elite kit, Vectorlabs, Burlingame, CA, USA) before being exposed to an avidin-biotinylated enzyme complex, stained with $\mathrm{DAB}$ and counterstained with hematoxylin.

\section{Protein analysis of markers of apoptosis and oxidative stress}

Protein expression of apoptotic and oxidative stress markers were measured in the cytosolic fraction collected for GR quantification. 20 $\mu$ g of total protein was loaded into $15 \%$ polyacrylamide gels and transferred to low fluorescence PVDF membranes and incubated overnight with the following apoptosis-related primary antibodies; BCL2 (1:1000, Abcam, Cat\#ab32124), BAX (1:000, Cell Signaling, Cat\#2772) and cleaved caspase 3 (1:1000, Cell Signaling, Cat\#9661) as well as primary antibodies for the antioxidant proteins thioredoxin reductase (Txnrd1, 1:1000, Abcam, Cat\#ab124954) and glutathione peroxidase (GPX, 1:1000, Abcam, Cat\#ab108427). After incubation with the primary antibody of interest, all membranes were incubated with a ACTB loading control (1:20,000, Cat\# A2228, Sigma Aldrich) for $1 \mathrm{~h}$. After careful washing, membranes were incubated with antimouse and anti-rabbit fluorescent secondary antibodies for $1 \mathrm{~h}$. Membranes were then scanned using the Licor Odyssey and densitometry determined using the 
supplied software. Total protein levels of the proteins of interest were normalized to the АСТВ loading control.

\section{Statistical analysis}

GR isoform data are presented as median, range and $\%$ expressed in Supplementary Table 1 (see section on supplementary data given at the end of this article). Through the remainder of the manuscript, all data are presented as mean \pm the standard error of the mean (s.E.M.). Statistical analysis was performed using GraphPad Prism 6 for Windows. For analysis of GR isoforms, three gels per sex were analyzed with a male cross gel calibrator used for the males and a female cross gel calibrator used for the females. As a different cross gel calibrator was used for males and females, all comparisons of GR isoforms between males and females are represented as ratios to total GR content. To analyse the effects of dexamethasone on GR isoform expression, all samples were normalized to the cross run calibrator for each sex. Unpaired $t$-tests were used to analyse the sex differences in GR isoform expression and to determine the differences in GR isoform expression compared to GR $\alpha$ A. T-tests were also used to compare the effects of saline and dexamethasone exposure on GR isoform expression, gene expression, expression of other proteins and protein carbonyl concentrations. When data did not fit Gaussian distribution, nonparametric analyses were performed (Mann-Whitney test). If data had unequal variance, an unpaired $t$-test with Welch's correction was applied. When normal analysis could not be performed due to the presence of zero values, data were transformed using $X=\log (X+0.1)$ before a nonparametric test was applied. $P<0.05$ was considered significant for all results.

\section{Results}

\section{Cytoplasmic GR expression in male and female saline-exposed (control) placentae}

Western blot analysis identified eleven GR bands corresponding to eight known isoforms and three unknown immunoreactive proteins (Fig. 1A). The known isoforms identified were GR $\alpha-\mathrm{A}(94 \mathrm{kDa}), \mathrm{GR} \alpha-\mathrm{B}(91 \mathrm{kDa})$, GR $\alpha-C(81 \mathrm{kDA})$ and GR $\alpha-\mathrm{D} 1-3(50-55 \mathrm{kDa})$ as well as the splice variants GRA ( $65 \mathrm{kDa})$ and GRP $(74 \mathrm{kDa})$. The three unknown bands identified were products at 69, 60 and $45 \mathrm{kDa}$, which have also been identified in other species (Saif et al. 2015, 2016). These unknown bands have not been confirmed as GR isoforms but are specific products
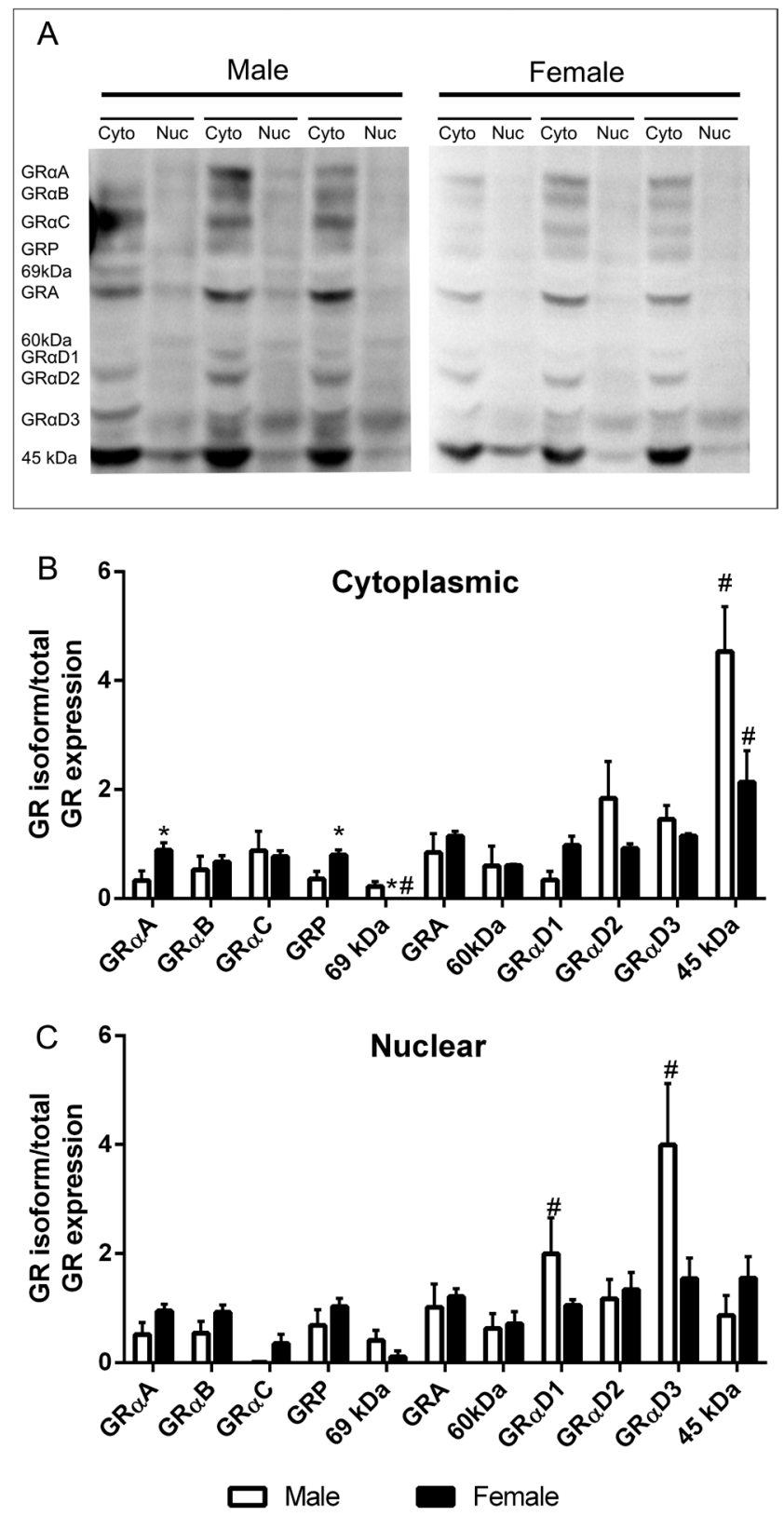

Figure 1

Representative Western blots of cytoplasmic (Cyto) and nuclear (Nuc) GR isoforms in placentae from saline-exposed male (open bars) and female (closed bars) mouse fetuses at embryonic day 14.5 (A). Densitometry was performed to measure the expression of each isoform for both the cytoplasmic (B) and nuclear (C) protein fraction. As protein levels of males and females were performed on different blots, data is presented as a ratio of each isoform to the total GR for each sample. Data are presented as mean \pm s.E.M., $n=8-12$ placentas from 10 litters per group, ${ }^{*} P<0.05$ represents sex difference in GR isoform, $\# P<0.05$ indicates that the GR isoform in question is different to GRo-A.

detected by this well-characterized antibody. The majority of isoforms (GR $\alpha-A, G R \alpha-B$, GR $\alpha-C$, GRP, GRA, GR $\alpha-D 2$, GR $\alpha$-D3) were expressed in the cytoplasmic fraction of 
all placentae investigated; however, the GR $\alpha$-D1 isoform and the protein bands corresponding to 69,60 and $45 \mathrm{kDa}$ were not always expressed (Supplementary Table 1). GRoD1 was expressed in 50\% of placentae from male fetuses but all placentae from female fetuses. The $69 \mathrm{kDa}$ protein was expressed in $83 \%$ of placentae from male fetuses but not expressed in any of the placentae from female fetuses. The $60 \mathrm{kDa}$ protein was expressed in the cytoplasmic protein extract in $67 \%$ of placentae from male fetuses but $100 \%$ from female fetuses. The $45 \mathrm{kDa}$ GR protein was expressed in the placentae of all male fetuses but only $80 \%$ of female fetuses. When each band was expressed as a ratio to total GR levels, the most highly expressed isoform detected was the unknown $45 \mathrm{kDa}$ protein, which was more highly expressed in both males and females compared to GR $\alpha-A(P<0.05$, Fig. 1B), whereas the $69 \mathrm{kDa}$ protein was lowly expressed being significantly less than GR $\alpha-A(P<0.05$, Fig. 1B). The protein expression of GR $\alpha-A$ and GRP were approximately 2-fold higher in placentae of females compared to males $(P<0.05$, Fig. 1B), whereas the $69 \mathrm{kDa}$ protein was lower in females than males $(P<0.05$, Fig. 1B).

\section{Nuclear GR expression in male and female saline-exposed (control) placentae}

GR $\alpha-A, G R \alpha-B$, GRP, GRA and GR $\alpha-D 1$ were found to be expressed in the nuclear fraction of all placentae (Supplementary Table 1). The GR $\alpha-C$ isoform was expressed in approximately $50 \%$ of nuclear fractions from placentae of female fetuses but only in $10 \%$ of placenta from male fetuses. In contrast, the $69 \mathrm{kDa}$ protein was expressed in $16 \%$ of placenta from female fetuses but in $66 \%$ of placentae from male fetuses. The $60 \mathrm{kDa}$ protein was expressed in the nuclear protein extract of all placentae from male fetuses but only $66 \%$ of placentae from female fetuses. The GR $\alpha$-D2 isoform expressed in $84 \%$ of placentae in both males and females and the GR $\alpha$ D3 isoform expressed in all male samples but in only $84 \%$ of female samples. Similarly, the $45 \mathrm{kDa}$ protein was expressed in all placentae from male fetuses but in $84 \%$ of placentae from female fetuses. GR $\alpha$-D3 was found to be the most highly expressed GR isoform in the nuclear fraction of placentae of male fetuses being significantly more highly expressed than the GR $\alpha$-A isoform $(P<0.05$, Fig. 1C). GR $\alpha-D 1$ was also significantly more highly expressed than the GR $\alpha-A$ isoform $(P<0.05$, Fig. 1C). $\mathrm{GR} \alpha-\mathrm{D} 3$ protein levels in placentae of female fetuses were found to be approximately 50\% of levels measured in placentae of male fetuses $(P=0.06$, Fig. $1 C)$.

\section{The effect of maternal dexamethasone infusion on placental GR isoform patterns}

Maternal dexamethasone exposure altered the expression of 6 different GR isoforms in a sex and cellular compartment-dependent manner (Fig. 2). Maternal dexamethasone exposure increased GR $\alpha$-A protein levels in the cytoplasmic fraction of placentae of male (Fig. 2C, $P<0.05)$ fetuses. In contrast, $\mathrm{GR} \alpha-\mathrm{C}$ protein levels were decreased in the cytoplasmic fraction of placentae of dexamethasone-exposed male fetuses (Fig. 2C, $P<0.05$ ). Maternal dexamethasone exposure had no effect on the expression levels of any GR isoform in the cytoplasmic fraction of placentae of female fetuses (Fig. 2D). Maternal dexamethasone exposure reduced the expression of nuclear GRA (Fig. 2E, $P<0.05$ ) but increased the expression of GR $\alpha-\mathrm{D} 2$ (Fig. 2E, $P<0.05$ ). Dexamethasone-exposed placentae of female fetuses had increased levels of GR $\alpha$-D1 and GR $\alpha$-D3 compared to placentae from saline-exposed fetuses (Fig. 2E, $P<0.05$ ). Maternal dexamethasone exposure tended to increase $\mathrm{GR} \alpha-\mathrm{C}$ expression in the nuclear fraction of placentae of female fetuses, but this did not reach statistical significance likely due to the fact that some samples in each group did not express this isoform.

\section{The effects of maternal dexamethasone infusion on placental mRNA expression of genes regulated by specific GR isoforms}

Given that the GR isoform profile was affected by maternal dexamethasone exposure in a sex-specific manner, mRNA levels of genes known to be regulated by specific GR isoforms were assessed. Dexamethasone exposure did not affect mRNA levels of Ier3 (regulated by all GR isoforms), Nfatc1 (regulated by GR $\alpha-A$ ), Bcl2l11 (regulated by GR $\alpha$ C) or Cpeb1 (regulated by GR $\alpha$-D3) in placentae of male fetuses (Fig. 3). $S g k 1$, a gene known to be regulated by GR $\alpha-C$, was increased by $53 \%$ in dexamethasone-exposed placentae of males compared to saline-exposed placentae of males (Fig. 3). In contrast, dexamethasone increased Ier3 by $58 \%$, reduced Nfatc1 by $34 \%$ and increased Bcl 2111 by $38 \%$ and $S g k 1$ by $96 \%$ in placentae of female fetuses compared to saline-exposed placentae (Fig. 3).

As nuclear translocation of the GR is an important mediator of GR signaling, expression of two factors known to regulate this process were examined. Maternal dexamethasone exposure increased Hspa $1 \mathrm{~b}$ mRNA levels in placentae of male $(P<0.05)$ but not female fetuses (saline male-1.06 \pm 0.11 , dexamethasone male- $1.64 \pm 0.23$, saline female-1.04 \pm 0.11 , dexamethasone female-1.18 \pm 0.12 )

Published by Bioscientifica Ltd. 

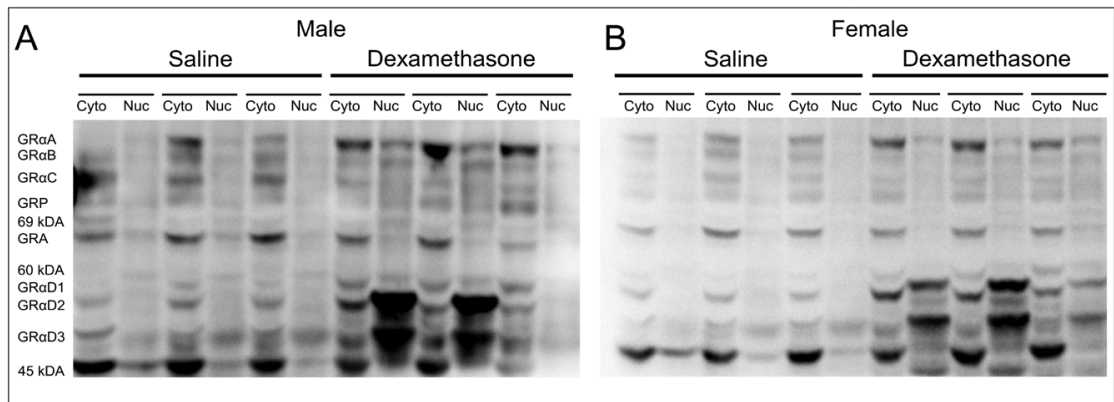

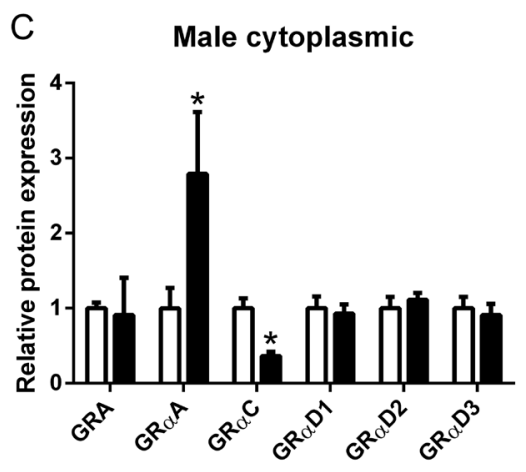

$\mathrm{E}$

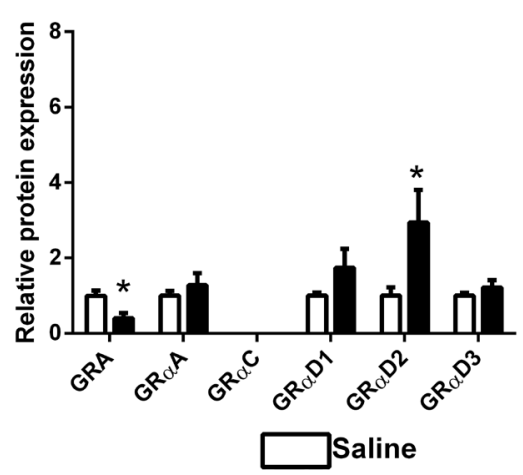

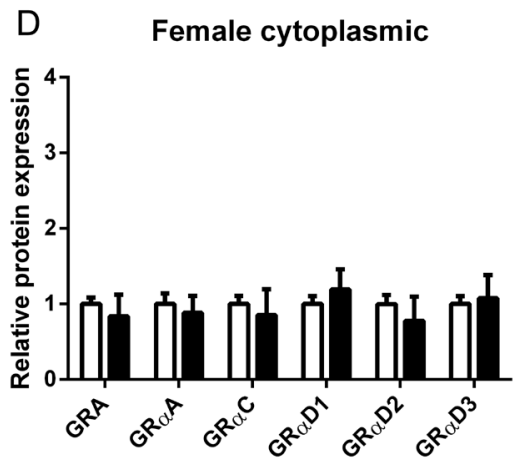

$\mathrm{F} \quad$ Female nuclear

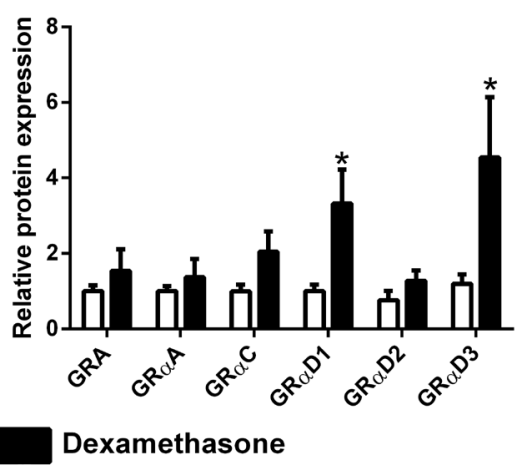

Figure 2

Representative Western blots of cytoplasmic (Cyto) and nuclear (Nuc) GR isoforms in saline (open bars) and dexamethasone (closed bars) exposed placentae from male (A) and female (B) mouse fetuses at embryonic day 14.5.

Densitometry was performed to measure the expression of each isoform for both the cytoplasmic (C and D) and nuclear ( $E$ and F) protein fractions. All samples from each sex were measured on three blots with a common sample on each membrane. As such total isoform expression was normalized to the average expression of the saline group for each isoform. Data are presented as mean \pm S.E.M., $n=8-12$ placentas from 10 litters per group, * $P<0.05$ represents significant effect of dexamethasone exposure. and had no effect on Ipo7 mRNA levels in either males or females (saline male-1.21 \pm 0.20 , dexamethasone male$1.51 \pm 0.26$, saline female-1.09 \pm 0.14 , dexamethasone female-1.08 \pm 0.08$)$.

\section{Effect of maternal dexamethasone exposure on placental apoptosis}

With female placentae having lower basal levels of the anti-apoptotic GR isoform GR $\alpha$-D3 and more frequently expressing the pro-apoptotic GR isoform GR $\alpha-C$ than males, we determined the impact of dexamethasone exposure on apoptotic factors in addition to $\mathrm{Bcl} 2 \mathrm{l11}$. This was particularly important given that dexamethasone reduced cytoplasmic expression of GR $\alpha-C$ in placentae of male fetuses. Cleaved caspase three immunostaining was only observed within the placental junctional zone (Fig. 4A, B, C and D). While staining was absent in salineexposed placentae (Fig. 4A and B), detectable staining was observed in placentae of dexamethasone-exposed male fetuses (Fig. 4C) and marked staining observed in placentae of dexamethasone-exposed female fetuses (Fig. 4D). While staining was not detected in all histological sections of placentas from male fetuses, marked staining was detected in all sections from female fetuses. Western blot analysis demonstrated that maternal dexamethasone exposure increased cleaved caspase-3 levels in placentae of both male and female fetuses (Fig. $4 \mathrm{E}$ and $\mathrm{F}, P<0.05$ ). In placentae of males, cleaved caspase-3 levels were increased by $55 \%$ but in females, the cleaved caspase-3 was increased by more than $250 \%$ compared to placentae of saline- 

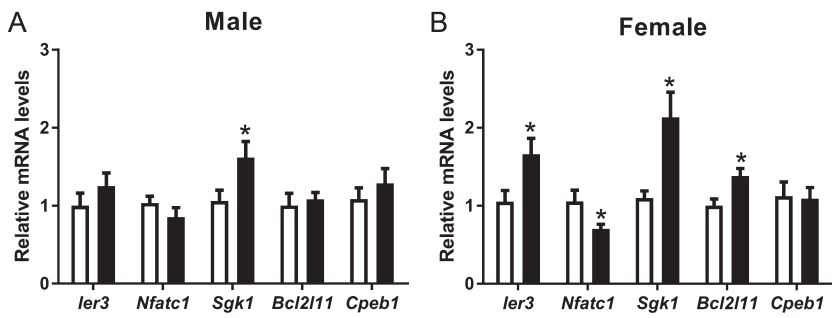

Figure 3

The effects of maternal dexamethasone (closed bars) on placental mRNA expression of target genes known to be regulated by GR isoforms in males (A) and females (B) compared to saline-exposed placentae (open bars). Data are presented as mean \pm S.E.M., $n=7-11$ placentas from 10 litters per group, ${ }^{*} P<0.05$ represents significant effect of dexamethasone exposure. ler3, immediate early response 3; Nfatc1 nuclear factor of activated T-cells 1; Sgk1, serum glucocorticoid kinase 1; Bcl2/11, Bcl-2-like protein 11; Cpeb1, cytoplasmic polyadenylation element-binding protein 1. ler3 is a marker of regulation by any GR isoform, Nfatc1 is regulated specifically by GR $\alpha-A, S g k 1$ and $B c / 2 / 11$ are regulated specifically by $\mathrm{GR} \alpha-\mathrm{C}$ and $C p e b 1$ is regulated by $\mathrm{GR} \alpha-\mathrm{D}$ isoforms.

exposed fetuses. Maternal dexamethasone had no effect on protein expression of BAX or BCL2 in placentae of either males (Fig. $4 \mathrm{G}$ and I) or females (Fig. $4 \mathrm{H}$ and $\mathrm{J}, P<0.05)$.

\section{Effect of maternal dexamethasone exposure on placental reactive oxygen species and antioxidant expression}

Glucocorticoid exposure can increase cellular oxidative stress, which can indirectly induce apoptosis. As such, this study measured placental protein carbonyl concentrations as a marker of oxidative stress, as well as protein levels of key antioxidants GPX1 and TXNRD1. Maternal dexamethasone exposure had no effect on placental protein carbonyl levels in males or females (Fig. 5A and B). Dexamethasone exposure increased protein expression of GPX and TXNRD1 in the placentae of males (Fig. 5C and E) but not females (Fig. 5D and F).

\section{Discussion}

This study has for the first time demonstrated the presence of multiple GR isoforms in the mouse placenta with eight known isoforms identified. In addition, we have identified three unknown proteins with GR immunoreactivity of similar size to those previously reported (Saif et al. 2015, 2016). Importantly, placentae of male and female mice have different GR isoform profiles that likely confer sexspecific cellular sensitivity to glucocorticoids. Of particular interest, nuclear expression of the anti-apoptotic isoform GR $\alpha$-D3 was greater in placentae of males compared to females while nuclear expression of the pro-apoptotic isoform $\mathrm{GR} \alpha-\mathrm{C}$ was higher in female placentae. This suggests that differences in placental glucocorticoid sensitivity between the sexes may be mediated by the differential expression of GR $\alpha-D$ and GR $\alpha-C$ isoforms and that apoptosis is likely to play a greater role in mediating the placental adaptations to glucocorticoid exposure in females than those in males.

The GR isoform profile of the murine placenta was similar to that in placentae of humans ( 8 known and five unknown) and guinea pigs (8 known and two unknown) (Saif et al. 2015, 2016), indicating analogous functional regulation of glucocorticoids between species. Indeed, using the same GR antibody, Orgeig and coworkers demonstrated that placental restriction in sheep reduces the expression of multiple GR isoforms in the fetal lung (Orgeig et al. 2015). Although GR was not measured in the placentas of these sheep, marked cellular and functional changes were noted in the placenta (Zhang et al. 2016). In our study in mice, GR $\alpha-C$ expression within the nuclear compartment was sex specific as was the expression of cytoplasmic isoforms such as GR $\alpha-A$ and GRP. In humans, placentae of male and female babies demonstrated sexspecific changes in $\mathrm{GR} \alpha-\mathrm{C}$ when born preterm compared to term while betamethasone exposure in guinea pigs also changed GR isoforms in a sex-specific manner. Despite similarities, there were key differences in GR isoform patterns between species. For example, GR $\alpha-C$ was not expressed at all in placentae of guinea pigs and instead other GR isoforms displayed sex-specific upregulation by glucocorticoid exposure (Saif et al. 2016). The most dominant isoform expressed also differed between species with GR $\alpha$-D1 being the most dominant isoform in the human placentae (Saif et al. 2015), but the $45 \mathrm{kDa}$ protein being the most highly expressed within the cytoplasmic compartment and GR $\alpha$-D3 the most highly expressed within the nucleus in mice.

Maternal dexamethasone exposure was also shown to affect GR isoform patterns within the placenta differentially in the cytoplasm and nucleus. The current study demonstrated that dexamethasone increased the expression of $\mathrm{GR} \alpha-\mathrm{A}$, but reduced the expression of GR $\alpha-C$ in the cytoplasmic fraction of the placental extracts of males but not females. These changes in GR isoform expression may mediate a range of non-genomic activities including regulation of chaperone proteins and altering cell signaling by interacting with MAPK/ERK/ AKT pathways (Mitre-Aguilar et al. 2015). Indeed, these glucocorticoid-induced changes in GR isoform expression levels may have contributed to the sex-specific regulation

Published by Bioscientifica Ltd 
Male
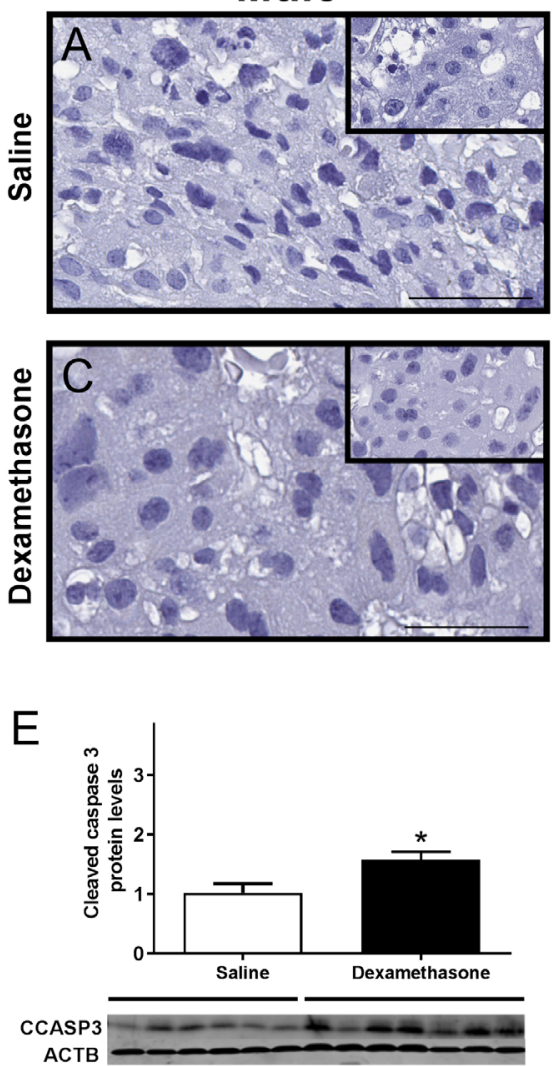

G
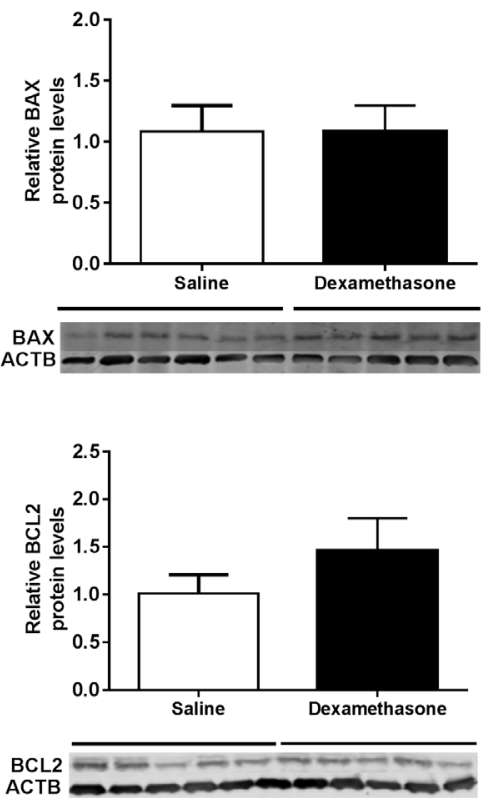

\section{Female}
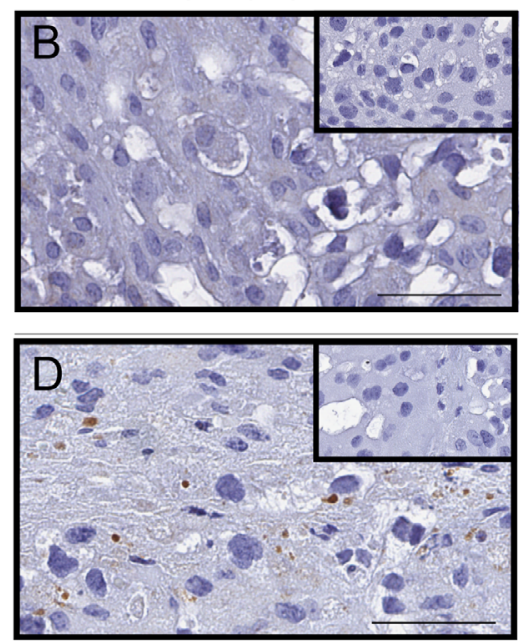

$\mathrm{F}$

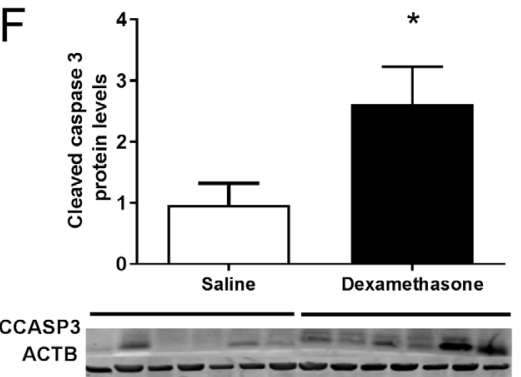

$\mathrm{H}$

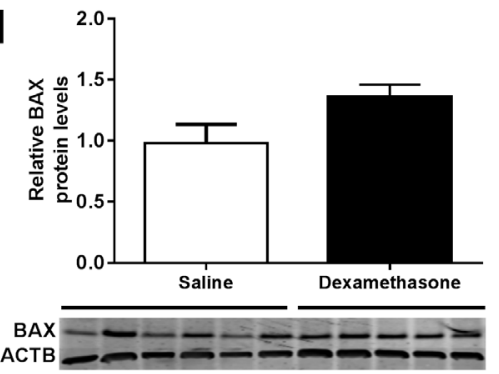

J

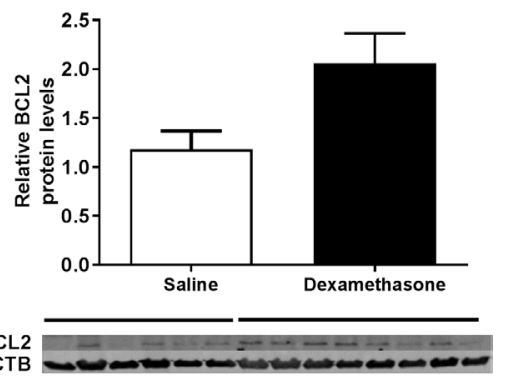

Figure 4

Immunohistochemical staining of cleaved caspase three (brown DAB pigment) in placentae of male ( $A$ and $C$ ) and female fetuses ( $B$ and $D$ ) exposed to saline ( $A$ and $B$ ) or dexamethasone ( $C$ and $D)$ during pregnancy ( $n=3$ per group). Isotype control sections are presented as inset images. All slides are counterstained with hematoxylin. The effects of maternal dexamethasone (closed bars) on placental cytoplasmic protein expression of cleaved caspase three in males (E) and females $(\mathrm{F}), \mathrm{BAX}$ in males $(\mathrm{G})$ and females $(\mathrm{H})$ and $\mathrm{BCL} 2$ in males (I) and females (J) compared to salineexposed placentae (open bars). Data is presented as mean \pm S.E.M., $* P<0.05$ represents significant effect of dexamethasone exposure. Representative blots contain $n=6$ out of the $n=8-12$ placentas from 10 litters per group analyzed. of MAPK previously demonstrated (Cuffe et al. 2011). In contrast, dexamethasone exposure had no effect on the expression of any GR isoforms within the cytoplasm of placentae of female fetuses suggesting that glucocorticoids may predominantly affect females through nuclear regulation of gene expression.

Nuclear expression of the $48 \mathrm{kDa}$ protein was increased with betamethasone treatment in human female http://joe.endocrinology-journals.org DOI: 10.1530/JOE-17-0171
๑) 2017 Society for Endocrinology Printed in Great Britain 


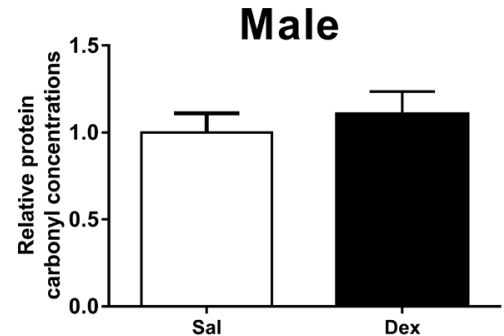

C

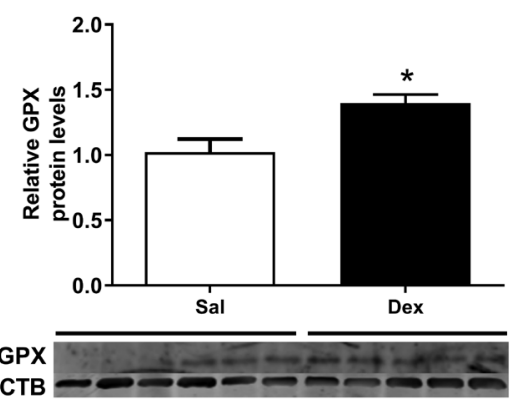

$E$

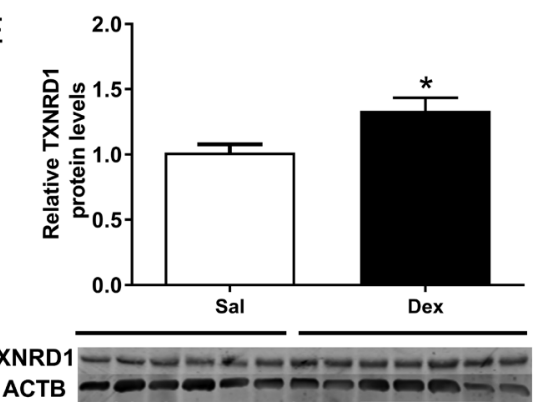

B

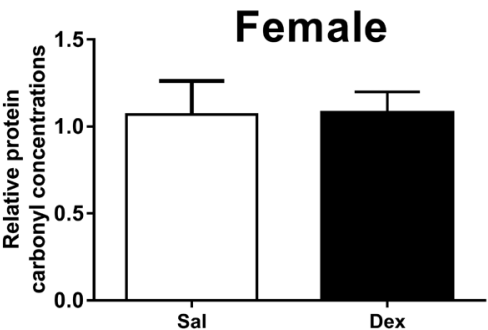

D
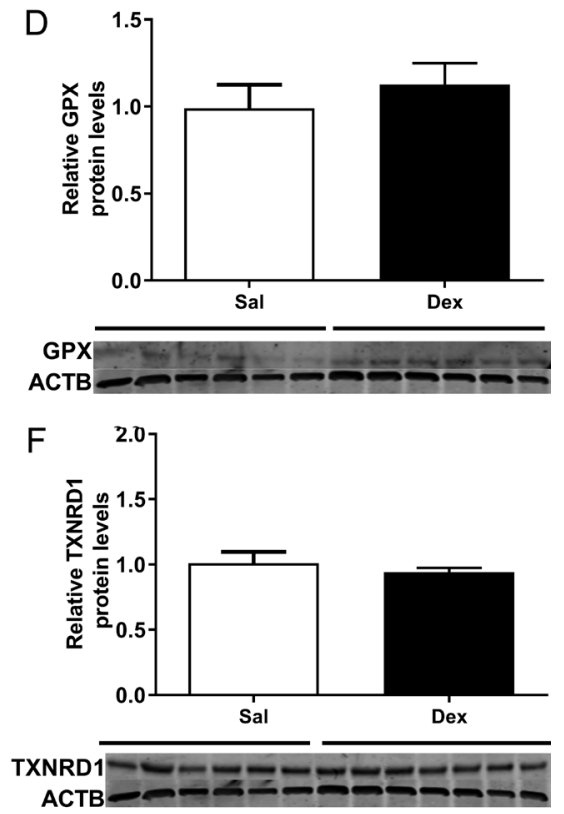

\section{Figure 5}

The effects of maternal dexamethasone (closed bars) on placental protein carbonyl levels in males (A) and females (B), placental GPX protein concentrations in males (C) and females (D) and TXNRD1 protein concentrations in males (E) and females (F) compared to saline-exposed placentae (open bars). Data is presented as mean \pm S.E.M., $* P<0.05$ represents significant effect of dexamethasone exposure.

Representative blots contain $n=6 . n=8-12$ placentas from 10 Litters per group were analyzed for Western blotting and protein carbonyl ELISAs. placentae but no others isoforms were affected in either sex (Saif et al. 2015). In guinea pig, preterm placentae exposed to betamethasone, there was an increase in GR $\alpha-A$ and GR $\beta$ in both sexes but male placentae had an increase in GR $\alpha$-D2 and D3 (Saif et al. 2016). The nuclear expression of GR isoforms in response to dexamethasone exposure in murine placental tissue is more complex with GRA being reduced and GR $\alpha$-D2 being increased in males while GR $\alpha$-D1 and 3 were increased in females. Each of these isoforms can function in the absence of ligand suggesting that these glucocorticoid-induced changes in nuclear GR isoform expression are not due to nuclear translocation but through other mechanisms. Furthermore, the transactivational activity of the GR $\alpha-D$ isoforms is lower than other GR isoforms suggesting that dexamethasone exposure has reduced glucocorticoid sensitivity by altering the expression of these isoforms. The cellular mechanisms responsible for dexamethasoneregulated GR isoform patterns are unclear but likely to be complex. Buoso and coworkers demonstrated that cortisol alters spliceosome proteins SRSF3 and SRSF9, which regulate the GR splice variants that are produced
(Buoso et al. 2017). This suggests that the dexamethasoneinduced regulation of the GR splice variant GRA in the current study may be due to altered spliceosomic activity. In addition, isoforms may have been affected by glucocorticoid-induced regulation of GR translation. Lu and Cidlowski previously demonstrated that ribosomal leaky scanning is responsible for $\mathrm{GR} \alpha-\mathrm{B}$ and $\mathrm{C}$ production, while ribosomal shunting is involved in GR $\alpha-C$ and $D$ formation ( $\mathrm{Lu} \&$ Cidlowski 2005). These processes may be regulated by epigenetic mechanisms with transcriptional microvariability thought to contribute to GR translational isoform profiles (Leenen et al. 2016). To date, no studies have investigated if glucocorticoids influence these processes but given our findings of changed GR $\alpha-C$ and $\mathrm{D}$ expression, these translational mechanisms may be involved.

Sex differences in glucocorticoid responsivity can regulate sex-biased gene expression profiles. A recent review proposes that glucocorticoid-induced GR expression in the placenta may mediate these gene profiles to affect fetal growth (Bivol et al. 2016). In mice, sex differences in GR levels mediate the dimorphic expression of inflammatory 
genes within the liver and deletion of the GR eliminated these sex differences (Quinn \& Cidlowski 2016). Differences in GR isoforms in dendritic cells have been shown to be responsible for the distinct gene profiles that change with maturity (Cao et al. 2013). Different populations of helper T-cells (TH1, TH2, TH17) express different GR isoforms, which determine GR sensitivity and regulate different patterns of glucocorticoid mediated gene expression (Banuelos \& $\mathrm{Lu}$ 2016). To date, these studies have highlighted the key differences in GR isoform regulation of apoptosis but there are undoubtedly other less well-characterized pathways affected in a sex- and tissue-specific manner.

Genes known to be regulated by different GR isoforms were similarly regulated by dexamethasone is a sex-specific manner in the mouse placenta. Ier3 (regulated by all GR isoforms) and Nfatc1 (Gro-A) were both affected by dexamethasone exposure in placentae from females but not males. Perhaps surprisingly, given that dexamethasone increased GR $\alpha$-D expression in both sexes, Cpeb1 (GR $\alpha$-D) mRNA levels were unaffected by dexamethasone in either sex. This might suggest that either Cpeb1 is not regulated by GR $\alpha-\mathrm{D}$ isoforms in placental cells or that the limited transactivational activity of GR $\alpha$-D protein prevented significant regulation of Cpeb1 expression. Sgk1 (GR $\alpha-\mathrm{C})$ was increased by dexamethasone but was increased to a much greater extent in females. This greater increase in Sgk1 further supports a more dominant role of GR $\alpha-C$ in the placental adaptations to glucocorticoids in females compared to males. Jurak cells transfected with GR $\alpha$-C have been shown to be highly vulnerable to dexamethasone-induced cell death (Wu et al. 2013) and the fact that GR $\alpha-C$ was only expressed in females in the current study is likely to have significantly influenced the regulation of apoptotic pathways. Indeed, Bcl2l11 has been shown to be regulated predominantly by GR $\alpha-C$ and in the current study was increased in the placentae of females but not males. Hspa1b, encodes HSP70, which along with HSP90 is an important component of the GR complex. While HSP90 promotes recovery of ligand binding, HSP70 inactivates the GR by partially unfolding the GR ligand-binding domain (Kirschke et al. 2014). Interestingly, Hspa $1 b$ mRNA levels were increased by $55 \%$ in dexamethasone-exposed placentae of male fetuses but did not change in placentae of female fetuses. This may further explain the observed increase in GR insensitivity in males compared to females and the glucocorticoid-induced increase in GR $\alpha$-A in the cytoplasmic compartment.
In our model, maternal dexamethasone administration lowers placental weight in female fetuses only (Cuffe et al. 2011). Morphological assessment demonstrated this was due to a reduction in the junctional zone area in females, a common placental adaptation to maternal stress (Coan et al. 2010, Mark et al. 2011). In response to a maternal challenge, the junctional zone has been shown to decrease in size to allow for expansion of the labyrinth (Burton \& Fowden 2012). The current study demonstrated cleaved caspase-3 staining only within the junctional zone. Western blotting analysis demonstrated that dexamethasone exposure increased cleaved caspase- 3 levels in both sexes; however, the increase was much greater in females. Previous studies have demonstrated that maternal dexamethasone exposure leads to apoptosis within the junctional zone of the rat placenta (Waddell et al. 2000, Ain et al. 2005); however, these studies did not investigate placentae of males and females separately. Apoptosis is also known to be initiated by oxidative stress. Restraint stress has been shown to increase oxidative stress levels in neurological tissues through endogenously produced glucocorticoids (Spiers et al. 2016). Furthermore, oxidative stress plays a key role in the etiology of many placental-related disorders (Jauniaux et al. 2006). We thus determined whether the apoptosis in the current study was a result of oxidative stress rather that direct GR signaling. This is of key interest given the GR is able to regulate expression of nuclear-encoded mitochondrial genes (Pgc1a and Nrf1) and activation of cytosolic GR receptors affect mitochondrial function (Lee et al. 2013). We found placental protein carbonyl levels were not affected by dexamethasone exposure in the mouse. Protein carbonyl levels are routinely used as markers of oxidative stress and are elevated in placental tissue of asthmatic women (Clifton et al. 2005). A range of antioxidants, including TXNRD1 and GPX, were also increased in asthmatic women independent of fetal sex. In the current study, TXNRD1 and GPX protein levels were increased in placentae of male but not female dexamethasone-exposed fetuses. This indicates that placentae of male fetuses may have additional glucocorticoid-regulated protective mechanisms against oxidative stress induced apoptosis in support of continued placental growth.

\section{Conclusion}

This study has for the first time demonstrated multiple GR isoforms in murine placenta. The pattern of GR

Published by Bioscientifica Ltd. 
isoforms expressed with the placenta is dependent on fetal sex and is regulated by exposure to glucocorticoids. Placentae of female fetuses were found to have greater levels of GR $\alpha$-C and lower GR $\alpha$-D, which may enhance the sensitivity to glucocorticoids and increase the degree of placental apoptosis compared to males. Importantly, however, this apoptosis was only increased within the junctional zone of placentae from female fetuses indicating that female fetuses are able to prioritize the labyrinth zone at this stage of gestation in response to a maternal challenge. Placentae of male fetuses continue to maximize their growth throughout this period of stress through the expression of the GR $\alpha$-D isoforms and via an alteration in GR protein configuration via HSP70 that reduces ligand binding. Apoptotic regulated reduction in junctional zone size in females may result in improved management of fetal resources which in turn contributes to the improved outcomes in later life for the female.

\section{Supplementary data}

This is linked to the online version of the paper at http://dx.doi.org/10.1530/ JOE-17-0171.

\section{Declaration of interest}

The authors declare that there is no conflict of interest that could be perceived as prejudicing the impartiality of the research reported.

\section{Funding}

J S M C was supported by a Griffith University research fellowship, K M M and V C L were supported by National Health and Medical Research Council Research Fellowships (V C L: APP1041918, K M M: APP1078164).

\section{Author contribution statement}

J S M C designed project, generated animals, performed most laboratory work and wrote most of the manuscript. Z S and J S M C performed G R isoform Western blots. A $\vee \mathrm{P}$ assisted with oxidative stress and antioxidant assessment and manuscript editing. K M M and V L C assisted with project design and manuscript editing.

\section{Acknowledgements}

The authors of this study would like to acknowledge those who contributed to this study but whose work did not warrant authorship. Thank you to Hayley Dickinson and Lee O'Sullivan and Emily Dorey for assistance with tissue collection, Sarah Steane for technical assistance and Oliva Holland for intellectual input.

\section{References}

Ain R, Canham LN \& Soares MJ 2005 Dexamethasone-induced intrauterine growth restriction impacts the placental prolactin family, insulin-like growth factor-II and the Akt signaling pathway. Journal of Endocrinology 185 253-263. (doi:10.1677/joe.1.06039)

Audette MC, Greenwood SL, Sibley CP, Jones CJ, Challis JR, Matthews SG \& Jones RL 2010 Dexamethasone stimulates placental system A transport and trophoblast differentiation in term villous explants. Placenta 31 97-105. (doi:10.1016/j.placenta.2009.11.016)

Banuelos J \& Lu NZ 2016 A gradient of glucocorticoid sensitivity among helper T cell cytokines. Cytokine and Growth Factor Reviews 31 27-35. (doi:10.1016/j.cytogfr.2016.05.002)

Bivol S, Owen SJ \& Rose'Meyer RB 2016 Glucocorticoid-induced changes in glucocorticoid receptor mRNA and protein expression in the human placenta as a potential factor for altering fetal growth and development. Reproduction, Fertility, and Development. (doi:10.1071/RD15356)

Braun T, Meng W, Shang H, Li S, Sloboda DM, Ehrlich L, Lange K, Xu H, Henrich W, Dudenhausen JW, et al. 2015 Early dexamethasone treatment induces placental apoptosis in sheep. Reproductive Sciences 22 47-59. (doi:10.1177/1933719114542028)

Buoso E, Galasso M, Ronfani M, Serafini MM, Lanni C, Corsini E \& Racchi M 2017 Role of spliceosome proteins in the regulation of glucocorticoid receptor isoforms by cortisol and dehydroepiandrosterone. Pharmacological Research 120 180-187. (doi:10.1016/j.phrs.2017.03.019)

Burton GJ \& Fowden AL 2012 Review: the placenta and developmental programming: balancing fetal nutrient demands with maternal resource allocation. Placenta 33 10. (doi:10.1016/ j.placenta.2011.11.013)

Cain DW \& Cidlowski JA 2015 Specificity and sensitivity of glucocorticoid signaling in health and disease. Best Practice and Research: Clinical Endocrinology and Metabolism 29 545-556. (doi:10.1016/j.beem.2015.04.007)

Cao Y, Bender IK, Konstantinidis AK, Shin SC, Jewell CM, Cidlowski JA, Schleimer RP \& Lu NZ 2013 Glucocorticoid receptor translational isoforms underlie maturational stage-specific glucocorticoid sensitivities of dendritic cells in mice and humans. Blood $\mathbf{1 2 1}$ 1553-1562. (doi:10.1182/blood-2012-05-432336)

Clifton VL 2010 Review: sex and the human placenta: mediating differential strategies of fetal growth and survival. Placenta 31 (Supplement) S33-S39. (doi:10.1016/j.placenta.2009.11.010)

Clifton VL, Vanderlelie J \& Perkins AV 2005 Increased anti-oxidant enzyme activity and biological oxidation in placentae of pregnancies complicated by maternal asthma. Placenta 26 773-779. (doi:10.1016/j.placenta.2004.10.018)

Coan PM, Vaughan OR, Sekita Y, Finn SL, Burton GJ, Constancia M \& Fowden AL 2010 Adaptations in placental phenotype support fetal growth during undernutrition of pregnant mice. Journal of Physiology 588 527-538. (doi:10.1113/jphysiol.2009.181214)

Cuffe JS, Dickinson H, Simmons DG \& Moritz KM 2011 Sex specific changes in placental growth and MAPK following short term maternal dexamethasone exposure in the mouse. Placenta 32 981-989. (doi:10.1016/j.placenta.2011.09.009)

Cuffe JS, O'Sullivan L, Simmons DG, Anderson ST \& Moritz KM 2012 Maternal corticosterone exposure in the mouse has sex-specific effects on placental growth and mRNA expression. Endocrinology 153 5500-5511. (doi:10.1210/en.2012-1479)

Cuffe JS, Steane S, Moritz KM \& Paravicini TM 2015 Differential mRNA expression and glucocorticoid-mediated regulation of TRPM6 and TRPM7 in the heart and kidney throughout murine pregnancy and development. PLOS ONE 10 e0117978. (doi:10.1371/journal.pone.0117978)

Eriksson JG, Kajantie E, Osmond C, Thornburg K \& Barker DJ 2010 Boys live dangerously in the womb. American Journal of Human Biology 22 330-335. (doi:10.1002/ajhb.20995) http://joe.endocrinology-journals.org

DOI: $10.1530 / J O E-17-0171$
() 2017 Society for Endocrinology Printed in Great Britain 
Fowden AL, Forhead AJ, Coan PM \& Burton GJ 2008 The placenta and intrauterine programming. Journal of Neuroendocrinology 20 439-450. (doi:10.1111/j.1365-2826.2008.01663.x)

Galon J, Franchimont D, Hiroi N, Frey G, Boettner A, EhrhartBornstein M, O'Shea JJ, Chrousos GP \& Bornstein SR 2002 Gene profiling reveals unknown enhancing and suppressive actions of glucocorticoids on immune cells. FASEB Journal 16 61-71. (doi:10.1096/fj.01-0245com)

Jauniaux E, Poston L \& Burton GJ 2006 Placental-related diseases of pregnancy: Involvement of oxidative stress and implications in human evolution. Human Reproduction Update 12 747-755. (doi:10.1093/humupd/dml016)

Kirschke E, Goswami D, Southworth D, Griffin PR \& Agard DA 2014 Glucocorticoid receptor function regulated by coordinated action of the Hsp90 and Hsp70 chaperone cycles. Cell 157 1685-1697. (doi:10.1016/j.cell.2014.04.038)

Lee SR, Kim HK, Song IS, Youm J, Dizon LA, Jeong SH, Ko TH, Heo HJ, Ko KS, Rhee BD, et al. 2013 Glucocorticoids and their receptors: insights into specific roles in mitochondria. Progress in Biophysics and Molecular Biology 112 44-54. (doi:10.1016/j.pbiomolbio.2013.04.001)

Leenen FA, Muller CP \& Turner JD 2016 DNA methylation: conducting the orchestra from exposure to phenotype? Clin Epigenetics 892. (doi:10.1186/s13148-016-0256-8)

Lu NZ \& Cidlowski JA 2005 Translational regulatory mechanisms generate $\mathrm{N}$-terminal glucocorticoid receptor isoforms with unique transcriptional target genes. Molecular Cell 18 331-342. (doi:10.1016/j.molcel.2005.03.025)

Mark PJ, Sisala C, Connor K, Patel R, Lewis JL, Vickers MH, Waddell BJ \& Sloboda DM 2011 A maternal high-fat diet in rat pregnancy reduces growth of the fetus and the placental junctional zone, but not placental labyrinth zone growth. Journal of Developmental Origins of Health and Disease 2 63-70. (doi:10.1017/S2040174410000681)

Mitre-Aguilar IB, Cabrera-Quintero AJ \& Zentella-Dehesa A 2015 Genomic and non-genomic effects of glucocorticoids: implications for breast cancer. International Journal of Clinical and Experimental Pathology 8 1-10.

O'Connell BA, Moritz KM, Roberts CT, Walker DW \& Dickinson H 2011 The placental response to excess maternal glucocorticoid exposure differs between the male and female conceptus in spiny mice. Biology of Reproduction 85 1040-1047. (doi:10.1095/ biolreprod.111.093369)

O’Regan D, Kenyon CJ, Seckl JR \& Holmes MC 2004 Glucocorticoid exposure in late gestation in the rat permanently programs genderspecific differences in adult cardiovascular and metabolic physiology. American Journal of Physiology: Endocrinology and Metabolism $\mathbf{2 8 7}$ E863-E870. (doi:10.1152/ajpendo.00137.2004)

O'Sullivan L, Cuffe JS, Paravicini TM, Campbell S, Dickinson H, Singh RR, Gezmish O, Black MJ \& Moritz KM 2013 Prenatal exposure to dexamethasone in the mouse alters cardiac growth patterns and increases pulse pressure in aged male offspring. PLOS ONE 8 e69149. (doi:10.1371/journal.pone.0069149)
Oakley RH \& Cidlowski JA 2013 The biology of the glucocorticoid receptor: new signaling mechanisms in health and disease. Journal of Allergy and Clinical Immunology 132 1033-1044. (doi:10.1016/j. jaci.2013.09.007)

Orgeig S, McGillick EV, Botting KJ, Zhang S, McMillen IC \& Morrison JL 2015 Increased lung prolyl hydroxylase and decreased glucocorticoid receptor are related to decreased surfactant protein in the growth-restricted sheep fetus. American Journal of Physiology: Lung Cellular and Molecular Physiology 309 L84-L97. (doi:10.1152/ ajplung.00275.2014)

Quinn MA \& Cidlowski JA 2016 Endogenous hepatic glucocorticoid receptor signaling coordinates sex-biased inflammatory gene expression. FASEB Journal 30 971-982. (doi:10.1096/fj.15-278309)

Saif Z, Hodyl NA, Stark MJ, Fuller PJ, Cole T, Lu N \& Clifton VL 2015 Expression of eight glucocorticoid receptor isoforms in the human preterm placenta vary with fetal sex and birthweight. Placenta 36 723-730. (doi:10.1016/j.placenta.2015.05.001)

Saif Z, Dyson RM, Palliser HK, Wright IM, Lu N \& Clifton VL 2016 Identification of eight different isoforms of the glucocorticoid receptor in guinea pig placenta: relationship to preterm delivery, sex and betamethasone exposure. PLOS ONE 11 e0148226. (doi:10.1371/ journal.pone.0148226)

Sharp AN, Heazell AE, Crocker IP \& Mor G 2010 Placental apoptosis in health and disease. American Journal of Reproductive Immunology 64 159-169. (doi:10.1111/j.1600-0897.2010.00837.x)

Singh RR, Cuffe JS \& Moritz KM 2012 Short- and long-term effects of exposure to natural and synthetic glucocorticoids during development. Clinical and Experimental Pharmacology and Physiology 39 979-989. (doi:10.1111/1440-1681.12009)

Spiers JG, Chen HJ, Cuffe JS, Sernia C \& Lavidis NA 2016 Acute restraint stress induces rapid changes in central redox status and protective antioxidant genes in rats. Psychoneuroendocrinology 67 104-112. (doi:10.1016/j.psyneuen.2016.02.005)

Vaughan OR, Sferruzzi-Perri AN \& Fowden AL 2012 Maternal corticosterone regulates nutrient allocation to fetal growth in mice. Journal of Physiology 590 5529-5540. (doi:10.1113/ jphysiol.2012.239426)

Waddell BJ, Hisheh S, Dharmarajan AM \& Burton PJ 2000 Apoptosis in rat placenta is zone-dependent and stimulated by glucocorticoids. Biology of Reproduction 63 1913-1917. (doi:10.1095/ biolreprod63.6.1913)

Wu I, Shin SC, Cao Y, Bender IK, Jafari N, Feng G, Lin S, Cidlowski JA, Schleimer RP \& Lu NZ 2013 Selective glucocorticoid receptor translational isoforms reveal glucocorticoid-induced apoptotic transcriptomes. Cell Death and Disease 4 e453. (doi:10.1038/ cddis.2012.193)

Zhang S, Barker P, Botting KJ, Roberts CT, McMillan CM, McMillen IC \& Morrison JL 2016 Early restriction of placental growth results in placental structural and gene expression changes in late gestation independent of fetal hypoxemia. Physiological Reports 4 e13049. (doi:10.14814/phy2.13060)

Received in final form 2 May 2017

Accepted 10 May 2017

Accepted Preprint published online 10 May 2017 http://joe.endocrinology-journals.org

DOI: 10.1530/JOE-17-0171 (c) 2017 Society for Endocrinology Printed in Great Britain
Published by Bioscientifica Ltd. 